\title{
Homing and movement patterns of a South African limpet Scutellastra argenvillei in an area invaded by an alien mussel Mytilus galloprovincialis
}

\author{
C. Ruiz Sebastián, C. N. Steffani, G. M. Branch* \\ Department of Zoology, Marine Biology Research Institute, University of Cape Town, Rondebosch 7701, South Africa
}

\begin{abstract}
Over the last three decades, the Mediterranean mussel Mytilus galloprovincialis has invaded rocky shores on the west coast of southern Africa, and now makes up $74 \%$ of intertidal mussel biomass. It competes directly with an abundant indigenous limpet, Scutellastra argenvillei, which attains high densities and biomass due to collective feeding on kelp. This competition has reduced the limpet's access to kelp, and dense aggregations of the limpets are now found only in small patches (termed 'established patches') that are remnants of the previous limpet belt. Lower densities of the limpets occur on mussel beds or in patches cleared of mussels by wave action ('cleared patches'). We investigated the effects of Mytilus encroachment on the movement patterns of $S$. argenvillei by comparing its homing and movements in these 3 situations. Short-term (12 h) homing frequency was similar on established patches (86.5\%) and newly created cleared patches $(81.3 \%)$ but significantly less on the mussel bed (60.4\%). On a monthly basis, homing frequency was lower, but significantly different in all 3 habitats (51.0, 29.2 and 10.9\%, respectively). Distances moved did not differ between habitats during the $12 \mathrm{~h}$ observations, but on a monthly basis were significantly greater on the mussel bed than in the other 2 habitats. There were no significant differences between the distances moved by juveniles versus adults for either $12 \mathrm{~h}$ or 1 mo periods. Small limpets on mussel beds frequently moved into cleared patches $(29.8 \%)$ while other limpets virtually never changed habitats. Mytilus encroachment has thus increased the mobility and reduced the homing frequency of $S$. argenvillei, probably because it limits access to kelp and increases dependency on alternative food sources. These changes in behaviour are associated with radical reductions of the density and mean size of $S$. argenvillei in areas dominated by $M$. galloprovincialis. Individuals that survive on the secondary substratum provided by mussels are all small and sexually immature. M. galloprovincialis has thus had an impact on both the behaviour and population dynamics of $S$. argenvillei, with considerable implications for the proposed commercial harvesting of $S$. argenvillei.
\end{abstract}

KEY WORDS: Homing $\cdot$ Limpet movement $\cdot$ Mussel encroachment $\cdot$ Scutellastra argenvillei $\cdot$ Mytilus galloprovincialis $\cdot$ Patellid limpet $\cdot$ Alien invasive

Resale or republication not permitted without written consent of the publisher

\section{INTRODUCTION}

Space is often the limiting resource in benthic marine systems, particularly on intertidal rocky shores (Branch 1984). On the west coast of southern Africa, recent invasion of rocky shores by an alien Mediter-

*Corresponding author. Email: gmbranch@egs.uct.ac.za ranean mussel, Mytilus galloprovincialis, has intensified competition for space (Grant \& Cherry 1985, Hockey \& van Erkom Schurink 1992). In particular, its domination of exposed to semi-exposed shores has brought it into direct competition with the limpet Scutellastra argenvillei (= Patella argenvillei: see Ridgway et al. 1998).

Scutellastra argenvillei is a large limpet, reaching 90 to $100 \mathrm{~mm}$, and occurs at high densities of up to $400 \mathrm{~m}^{-2}$, 
forming a conspicuous belt in the lower eulittoral zone of the northern west coast, termed the Argenvillei zone (Stephenson 1939). The high density and biomass achieved by $S$. argenvillei are consequences of specialised feeding behaviour and subsidy by subtidal kelp (Bustamante et al. 1995). Adults are sedentary and occupy well-developed home scars (Branch 1971). When blades of the kelps Ecklonia maxima or Laminaria pallida are swayed within reach by the incoming tide, adult limpets trap the blades between the shell edge and the substratum, and then feed collectively on them. These peculiar feeding habits result in a markedly sedentary life-style and a high degree of homing among adults, which rely on capturing food brought to them instead of actively foraging. This mode of feeding is also associated with the formation of limpet aggregations, as the chances of collective feeding increase with increasing limpet density. Less information is available on the diet of juvenile and subadult limpets, but Bustamante et al. (1995) suggested that small limpets ( $<50 \mathrm{~mm}$ shell length) seldom feed on kelp but rather on epilithic micro-algae.

Mytilus galloprovincialis only became established on the west and south coasts of southern Africa in the last 3 decades. Currently, it covers over $2000 \mathrm{~km}$ of the coast and has become the dominant mussel species, contributing an estimated $74 \%$ of intertidal mussel biomass (Grant \& Cherry 1985, van Erkom Schurink \& Griffiths 1990, Griffiths et al. 1992). M. galloprovincialis similarly dominates the southern coast of California and has apparently competitively displaced the indigenous $M$. trossulus (Geller 1999). It has also invaded the British Isles, Japan and Hong Kong (McDonald \& Koehn 1988, Geller 1999). In the intertidal zone of the west coast of South Africa, M. galloprovincialis outcompetes the local mussels Aulacomya ater and Choromytilus meridionalis because of its higher growth rate, superior reproductive output and higher tolerance to desiccation. It also displaces adult Scutellastra (= Patella) granularis from primary space, although its beds provide better settlement and recruitment sites for juveniles of this limpet than do those of A. ater (Hockey \& van Erkom Schurink 1992).

Competitive interactions between sessile species and limpets are not unusual. Branch (1976) noted that the barnacles Chthamalus dentatus, Octomeris angulosa and Tetraclita serrata compete with Scutellastra granularis for space and deny it access to food, resulting in reduced limpet size, growth and reproductive output. Lewis \& Bowman (1975) found similar effects on Patella vulgata in interactions with Mytilus edulis and Semibalanus balanoides. In Australia, dense stands of barnacles exclude the limpet Cellana tramoserica (Creese 1982). Interactions between limpets and sessile organisms can, however, be complex. For example, Iwasaki (1993b) showed that grazing by the limpet Cellana toreuma depletes foliose algae and thereby promotes settlement of barnacles. The development of barnacle stands, however, excludes $C$. toreuma, and if $C$. toreuma is the only limpet present, foliose algae then proliferate and smother the barnacles.

Long-term observations on the intertidal zone of Namaqualand on the west coast of South Africa have shown that Mytilus galloprovincialis has expanded over the last 20 yr (G. M. Branch unpubl. data, Eekhout et al. 1992) and has now substituted previously dense aggregations of Scutellastra argenvillei, particularly on shores exposed to strong wave action. What was once an extensive limpet belt had been reduced to small limpet patches interspersed in the mussel bed, although the mussel bed itself is occupied by limpets. As filter-feeders, mussels do not compete with limpets for food. They do, however, occupy primary space that could otherwise be used by limpets, and reduce the limpets' access to food. Exploitative competition for primary space is thus the main interaction between these species. M. galloprovincialis invades gaps in its beds by lateral expansion, and larval settlement seems to occur directly into mussel beds or crevices, rather than on bare rock (N. Steffani unpubl. data). At Tatoosh Island in Washington, USA, patches smaller than $3 \mathrm{~m}^{2}$ that are created in beds of $M$. californianus disappear due to lateral encroachment by peripheral mussels (Paine \& Levin 1981). Similar lateral expansion occurs in beds of Semimytilus algosus (Tokeshi \& Romero 1995). In the case of M. galloprovincialis, high densities of adult $S$. argenvillei or $S$. granularis retard invasion of primary rock space by $M$. galloprovincialis (Eekhout et al. 1992, Hockey \& van Erkom Schurink 1992).

Homing behaviour and movement patterns have been extensively studied for a range of limpet species (reviewed by Branch 1981, Hartnoll 1985, Chapman \& Underwood 1992) and for other gastropods and chitons (reviewed by Underwood 1979, Hawkins \& Hartnoll 1983, Little 1989, Chelazzi 1990). Homing clearly has different functions in different species, including avoidance of desiccation (Branch \& Cherry 1985, Iwasaki 1994), reduction of predation (Branch 1978, Garrity \& Levings 1983, Iwasaki 1992, 1993a), survival of wave action (Branch 1988, Gray \& Hodgson 1998), defence of a territory or maintenance of dominance (Stimson 1970, Branch 1975, Iwasaki 1995) and reduction of intraspecific competition (Mackay \& Underwood 1977, Iwasaki 1995). Most evidence suggests that limpets find their way home by following chemical trails laid down on the outward trip, although there may be other mechanisms (Funke 1968, Cook et al. 1969, Branch 1981, Chelazzi et al. 1988). Intrapopulation differences in homing have been noted (e.g. 
Mackay \& Underwood 1977, Underwood 1977, Iwasaki 1993c, 1994, Serra et al. 2001), and Iwasaki (1999) has specifically addressed how limpet homing and movement patterns are affected by interactions with sessile species. Scutellastra argenvillei homes to 'scars' (depressions eroded in the rock or on the shells of conspecifics), but these are poorly defined on hard rocks (Branch 1971). No home scars have been observed when the limpets occupy mussels, although small limpets form obvious scars on the shells of larger individuals of $S$. argenvillei.

The final impact of Mytilus galloprovincialis on populations of Scutellastra argenvillei is likely to be determined by a combination of abiotic factors, including wave action, shore height and topographic complexity, and biotic factors such as rates of lateral expansion and larval settlement by mussels, and limpet size, mobility, grazing and bulldozing. In this study, we addressed the effects that M. galloprovincialis has on the population structure and movement patterns of $S$. argenvillei on 3 different types of habitats: (a) 'established patches' of $S$. argenvillei (remnants of the original natural limpet belt, where limpets still aggregate at high densities), (b) 'cleared patches' (gaps in the mussel bed created by wave action and later colonised by $S$. argenvillei at relatively low densities), and (c) mussel beds. Comparisons were made of homing and movement patterns over short-term (12 h) and medium-term (1 mo) intervals, between limpets of 2 size classes (small and large), and between diurnal and nocturnal periods. Mussels present an irregular and unstable substratum and are likely to hinder feeding of limpets, particularly adult limpets that derive a significant portion of their food by trapping kelp. As a result, we expected limpets to home less frequently and move greater distances when they are on mussels than when attached to rock in established patches, and that adult limpets would move less than juveniles because of their lower mass-specific metabolic rate and their dependence on kelp blades. We hypothesised that (1) homing will be most frequent on established patches, intermediate on cleared patches and least frequent on mussel beds; (2) distances moved will be greatest on mussel beds, intermediate on cleared patches and least on established patches; (3) adult limpets will move less frequently and shorter distances than juveniles; (4) the frequency at which limpets move from mussel beds to cleared or established patches will be greater than in the opposite direction.

\section{MATERIALS AND METHODS}

The study area was located at Groenrivier $\left(30^{\circ} 50^{\prime} \mathrm{S}\right.$, $\left.17^{\circ} 40^{\prime} \mathrm{E}\right)$ on the west coast of South Africa, where
Mytilus encroachment on Scutellastra argenvillei has been monitored for over a decade (G. M. Branch unpubl. data). The area is isolated from any major human settlement and thus largely undisturbed, and is currently under consideration as a new National Park. The study site, Island Wreck, was located $12 \mathrm{~km}$ south of the Groenrivier mouth and consists of a $30 \mathrm{~m}$ stretch of west-facing wave-exposed rocky shore, occupied mainly by mussel beds of $M$. galloprovincialis, which covered about the lower $5 \mathrm{~m}$ of the shore. Such rocky shores are fringed with subtidal forests of kelp (Ecklonia maxima and Laminaria pallida), and support dense colonies of $S$. argenvillei and beds of M. galloprovincialis (Bustamante et al. 1995). Tidal range was $1.9 \mathrm{~m}$ and the mussel beds occupied the lower half of the tidal range. Sampling of limpet movement was confined to the lowermost $50 \mathrm{~cm}$, where $S$. argenvillei is concentrated. For our study, 3 clearly distinguishable habitats were recognised in this zone: 'mussel beds' with virtually continuous multi-layered mussels covering 80 to $90 \%$ of the substratum, 'established patches' (bare rock with highdensity aggregations of $S$. argenvillei that are remnants of the original limpet belt and now form distinct patches spanning 700 to $5000 \mathrm{~cm}^{2}$ in area), and 'cleared patches' (rock cleared of mussels by wave action and now housing remains of mussel beds with variable limpet densities, occupying irregular areas of 400 to $10000 \mathrm{~cm}^{2}$ ). Established patches and mussel beds were relatively homogeneous habitats. By contrast, cleared patches comprised a range of conditions from recently cleared areas with a mix of colonisers, through different successional stages of ephemeral algae and grazers, to grazer-dominated bare rock with encrusting algae. As an indication of the homogeneity of the 3 habitats, ten $0.25 \mathrm{~m}^{2}$ quadrats were randomly positioned in each habitat, and the percentage covers of the 3 most abundant taxa ( $M$. galloprovincialis, Porphyra sp. and coralline turf) scored in each quadrat. These values were then used to calculate the average coefficient of variation for all 3 taxa, which was used as an index of the biotic homogeneity of each habitat.

Between 55 and 90 Scutellastra argenvillei were tagged in a number of areas in each habitat (see Table 1 for sample sizes). Tagging was limited to limpets $>20 \mathrm{~mm}$ in length, and included a representative sample of both juveniles ( 20 to $49 \mathrm{~mm}$ ) and adults ( $>50 \mathrm{~mm}$ ), because individuals less than $50 \mathrm{~mm}$ seldom feed on kelp whereas most large individuals do (Bustamante et al. 1995). At 7 randomly distributed points, pairs of reference points were established parallel to the shore, their distance apart measured, and their positions marked with nails hammered into holes drilled into the rock. 
Table 1. Summary of the numbers of small (20-49 $\mathrm{mm})$ and large (>50 $\mathrm{mm}$ ) Scutellastra argenvillei that were tagged and their percentage recovery after 2 mo

\begin{tabular}{|lccc|}
\hline & \multicolumn{2}{c}{ Limpets } & \multirow{2}{*}{$\begin{array}{c}\text { Recovery } \\
\text { nynn }\end{array}$} \\
\cline { 2 - 3 } & Tagged & Recovered & \\
\hline Established patches & 55 & 52 & 94.5 \\
Small limpets & 19 & 17 & 89.5 \\
Large limpets & 36 & 35 & 97.2 \\
Cleared latches & 61 & 56 & 91.8 \\
Small limpets & 37 & 35 & 94.6 \\
Large limpets & 24 & 21 & 87.5 \\
Mussel beds & 90 & 73 & 81.1 \\
Small limpets & 73 & 62 & 84.9 \\
Large limpets & 17 & 11 & 64.7 \\
Total & 206 & 181 & 87.9 \\
\hline
\end{tabular}

Limpets were measured and tagged at low water without removing them from the substratum, using standard quick-setting Pratleys putty and a numerical serial number on a coloured plastic adhesive tape (Dymo tape), as in previous studies. Encrusting material on the limpets' shells was removed with a soft wire brush prior to application of the putty, to facilitate adhesion. For the smallest limpets, a bee-tag was embedded in a small disk (6 to $7 \mathrm{~mm}$ ) of clear glue instead of using Dymo tape, because of the risk that the Dymo tape labels and putty would hinder the movements of small limpets. The positions of limpets were recorded by measuring the distances from the apex of each limpet's shell to each of the 2 fixed reference points $\left(D_{1}, D_{2}\right)$, and noting whether the limpet was positioned seawards or landwards of a line between the reference points.

The positions of limpets and the habitats on which they occurred were monitored during low spring tides between March and May 1999, when the tidal excursion was predicted to be greatest, thus allowing easiest access to the low shore. Monitoring occurred every $12 \mathrm{~h}$ for 4 successive monitoring sessions in March, 7 in April and 1 in May. Movements recorded at $12 \mathrm{~h}$ intervals were classed as 'short-term' and those at monthly intervals as 'medium-term'.

The different sampling periods were used for different purposes. In total, 206 limpets were tagged. For an assessment of recovery rates of large and small limpets in the 3 habitats, numbers of tagged animals were compared at the start and after 2 mo. For the measurements of homing frequency and distances moved, only one $12 \mathrm{~h}$ period and one 1 mo period were selected, to avoid non-independence of data (repeated measures on the same limpets on successive days). For the same reason, differences between day and night movements were based on the data for a single day. The periods selected were those that yielded the largest data sets. The calculations of distances moved were based on only those limpets that did move, thus excluding those that remained in their original positions. To measure the frequencies with which individual limpets moved between successive periods of observation, the entire data set was employed, as these data were independent. Finally, to quantify movements between habitats, the habitats originally occupied by limpets were compared with those they occupied 1 mo later.

The distances limpets moved were calculated using polar geometry, following a method similar to that of Underwood (1977). Distance measurements from the reference points $\left(D_{1}, D_{2}\right.$, up-shore or down-shore position) were converted into polar co-ordinates by triangulation, with point of origin being defined as the northernmost reference point. Reference markers were chosen to be co-planar with the limpet movement plane. Short- and medium-term distances moved by limpets were calculated by triangulation using the corresponding polar co-ordinates.

To obtain a measure of the error inherent in the methods, laboratory simulations were employed. Three limpet shells were positioned at different distances and angles from 2 reference points and moved to obtain 40 observations. Direct measurements of moved distance were compared with distances calculated by triangulation. The mean difference between measured and estimated distance was $0.65 \mathrm{~mm}$ (SD $11.0 \mathrm{~mm}$ ) and the maximum difference was $28 \mathrm{~mm}$. It was therefore concluded that distances of less than $30 \mathrm{~mm}$ could not be accurately considered displacement, since the average size of the limpets was greater than this. Limpets were considered to be 'homing' during any given period when they moved $<30 \mathrm{~mm}$, regardless of the presence of a home-scar or the degree of movements over other time periods. Calculations such as these, being based on the displacement of limpets over time intervals, will always underestimate the actual distances moved as they do not trace the paths of movement (Erlandsson et al. 1999). They have, however, been widely used as valid relative measurements of movement and homing.

Frequencies of homing of small and large limpets were compared for each habitat separately using $\chi^{2}$-tests. Frequencies of homing in different habitats were analysed, using another $12 \mathrm{~h}$ data set, with $\chi^{2}$-tests followed by Tukey-type post hoc tests described in Zar (1999). Distances moved were compared between habitats (mussel beds, cleared and established patches) and sizes (small versus large limpets) with 2-way ANOVA, with both habitat and size being fixed factors. Post hoc tests (Tukey's honestly significant difference for unequal $n$ ) were used to examine the nature of differences deemed significant by ANOVA tests. 
Prior to the analysis, the data were tested for normality (Kolmogorov-Smirnov test) and for homogeneity of variances (Levene's test) and if necessary transformed to meet statistical assumptions (Underwood 1997, Zar 1999). For comparisons of distances moved during the day versus night, normality of data could not be achieved. Therefore, we used a Wilcoxon's pairedsample test, pairing day and night movements for individual limpets (Zar 1999, p. 165). Frequencies of changes between habitats were analysed with $\chi^{2}$-tests followed by Tukey-type post hoc tests (Zar 1999, p. 563). The densities of $S$. argenvillei in each of the 3 habitats were determined from 5 samples of $0.25 \mathrm{~m}^{2}$ in each habitat and their sizes from measurements (to the nearest $\mathrm{mm}$ ) of the first 50 limpets encountered in each habitat. Comparisons between habitats were based on 1-way ANOVAs, with habitat as a fixed factor, followed by Tukey tests. Unless otherwise stated, all analyses were carried out using Statistica (1999, version 5.5).

\section{RESULTS}

In total, 206 Scutellastra argenvillei were tagged, 181 of which were recaptured after 2 mo. Recovery rates were high, averaging $87.9 \%$, but were lowest for large limpets on mussel beds $(64.7 \%)$ and highest for large limpets on established patches $(97.2 \%$; Table 1$)$.

The sizes of tagged limpets ranged between 24 and $77 \mathrm{~mm}$, with a higher percentage of small limpets on mussel beds than in the other habitats (Table 1), and a lower percentage of large limpets on mussel beds. Although limpets $>50 \mathrm{~mm}$ were actively sought for on mussel beds, only 17 were found.
One-month homing frequencies were lower than the short-term homing frequencies in all size-habitat categories (Fig. $1 ; \chi^{2}$-tests, $\mathrm{p}<0.01$, all cases). On average, $71.3 \%$ of the limpets homed during the $12 \mathrm{~h}$ observations, whereas only $23.8 \%$ remained in the same position after $1 \mathrm{mo}$. None of the large limpets on mussel beds remained at the same position 1 mo later, and $>87 \%$ of the small limpets in this habitat moved. One-month homing frequencies on mussel beds were lower than in either of the other habitats $\left(\chi^{2}=22.16, \mathrm{df}=2, \mathrm{p}<0.01\right.$; Tukey-type tests, $\mathrm{p}<0.01$ and $<0.05$ for established and cleared patches, respectively), and homing of limpets in cleared patches was significantly less frequent than in the established patches $(p<0.05)$. No differences in 1 mo homing frequencies were found between small and large limpets $\left(\chi^{2}\right.$-tests, $p>0.1$ in all habitats).

In short, homing was always less frequent on mussels than on the other 2 habitats, but did not differ between small and large limpets. These patterns were consistent all 12 times on which $12 \mathrm{~h}$ observations were made and both times 1 mo comparisons were made, although the statistical analyses were confined to one $12 \mathrm{~h}$ and one 1 mo period to avoid non-independence of the data.

\section{Frequency of limpet movements}

The frequencies with which individual limpets moved from their previously recorded positions were recorded over eight $12 \mathrm{~h}$ periods ( 3 consecutive periods in March and 5 in April). Only limpets recorded more than 4 times in the sampling period were taken into

\section{Homing}

During the $12 \mathrm{~h}$ short-term observations (Fig. 1A), small limpets in established patches showed the highest frequency of homing $(94.1 \%)$ and large limpets on mussel beds the lowest $(50.0 \%)$. Limpets on mussel beds homed less frequently than those on either cleared or established patches $\left(\chi^{2}=11.32, \mathrm{df}=2, \mathrm{p}<0.01\right.$; Tukey-type tests: $\mathrm{p}<0.05$ and $<0.01$, respectively), but there was no difference between the latter 2 habitats $(p>0.05)$. There was no significant difference between the frequencies of homing for large versus small limpets in any of the habitats $\left(\chi^{2}\right.$-tests, $\mathrm{p}>0.37$ in all comparisons).
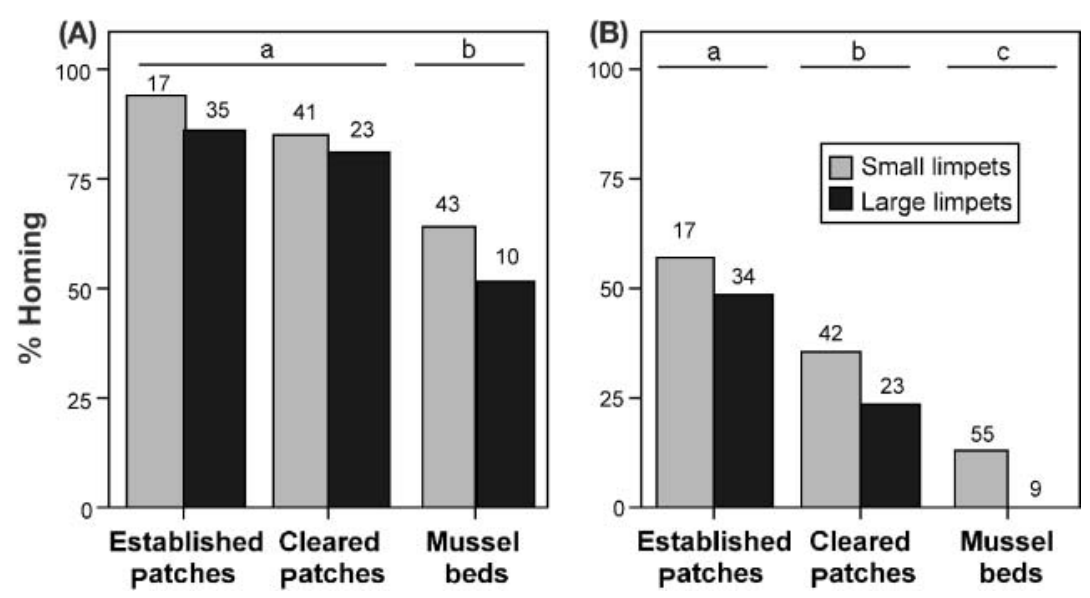

Fig. 1. Scutellastra argenvillei. (A) Short- (12 h) and (B) medium-term (1 mo) percentage homing of in different habitats. Numbers above the bars refer to the sample size. Horizontal lines with different letters indicate significant differences between habitats. There were no significant differences between small and large limpets (see text for details) 

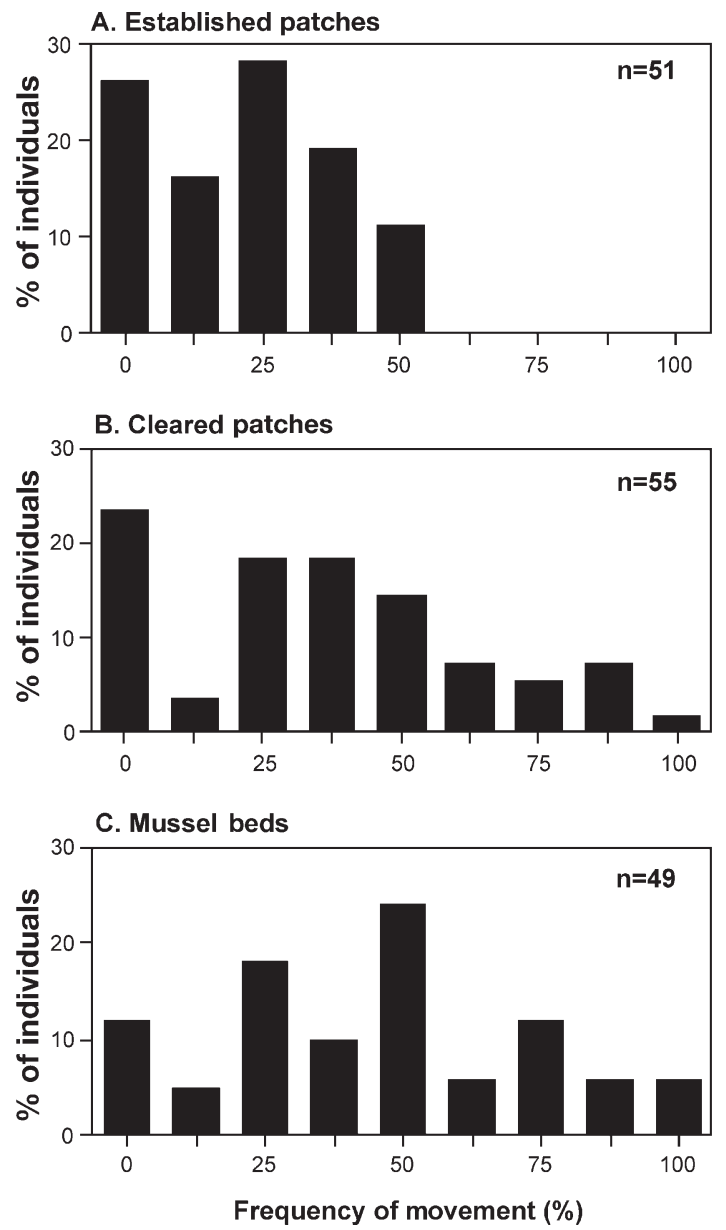

Fig. 2. Scutellastra argenvillei. Frequency of movement in different habitats, measured over 8 consecutive $12 \mathrm{~h}$ periods. Frequency of movement is expressed as the percentage of 12 h observation periods during which movement occurred, and ranges from $0 \%$ (when a limpet did not change position in any $12 \mathrm{~h}$ period) to $100 \%$ (when a limpet changed position every $12 \mathrm{~h}$ period)

account. Limpets in established patches usually did not move $(25 \%)$ or moved only once $(36.5 \%)$, and none changed location more frequently than $50 \%$ of the time (Fig. 2). On mussel beds, the modal frequency of movement was $50 \%$, although the frequency spanned the full range from 0 to $100 \%$. An intermediate pattern appeared in cleared patches, where a substantial proportion of the limpets $(23.6 \%$ ) did not move, while $35 \%$ moved at least half of the time.

\section{Distances of movements}

Comparisons showed that the distances moved by day and by night were never significantly different (Wilcoxon paired-sample tests: $\mathrm{n}=52, \mathrm{p}=0.25 ; \mathrm{n}=62$, $\mathrm{p}=0.44 ; \mathrm{n}=48, \mathrm{p}=0.21$, for established patches, cleared patches and mussel beds, respectively).

Measurements of distances moved over $12 \mathrm{~h}$ periods were only tested for the effect of habitat since the size classes had to be pooled due to low number of observations in some of the categories (e.g. small limpets in established patches; Fig. 3A). Habitat had no significant effect (ANOVA, $F_{2,37}=0.118, \mathrm{p}=0.89$ ). The effect of limpet size on distance moved was explored directly by linear regression of log-transformed distance on limpet size. This showed that the relationship was not significant in any of the habitats $\left(\mathrm{r}^{2}=0.03, \mathrm{df}=\right.$ $5, \mathrm{p}=0.72 ; \mathrm{r}^{2}=0.14, \mathrm{df}=10, \mathrm{p}=0.22 ; \mathrm{r}^{2}=0.05, \mathrm{df}=19$, $\mathrm{p}=0.30$ for established patches, cleared patches and mussel beds, respectively).

Over 1 mo, 2-way ANOVA was used to test for the effects of size and habitat on distances moved (Table 2, Fig. 3B). There was no significant interaction between these factors. Limpets in different habitats moved different amounts. Tukey tests showed significant differences $(p<0.01)$ between limpets on mussel beds (which were the most mobile, averaging $38.4 \pm 4.0 \mathrm{~cm}$ ) and those on cleared patches $(23.1 \pm 4.4 \mathrm{~cm})$ or on established patches $(13.1 \pm 1.8 \mathrm{~cm})$. Limpets in the latter 2 habitats did not differ significantly (Tukey test, $\mathrm{p}=0.24$ ).

Size class had no significant influence on distance moved per month (Table 2). The relationship between limpet size and distance moved was investigated further using linear regressions of log-transformed 1 mo distances on limpet size. These showed no significant relationship in the cases of established patches and mussel beds $\left(\mathrm{r}^{2}=0.11, \mathrm{df}=23, \mathrm{p}=0.11\right.$ and $\mathrm{r}^{2}=0.05$, $\mathrm{df}$ $=55, \mathrm{p}=0.11$, respectively), but did detect a weak rela-
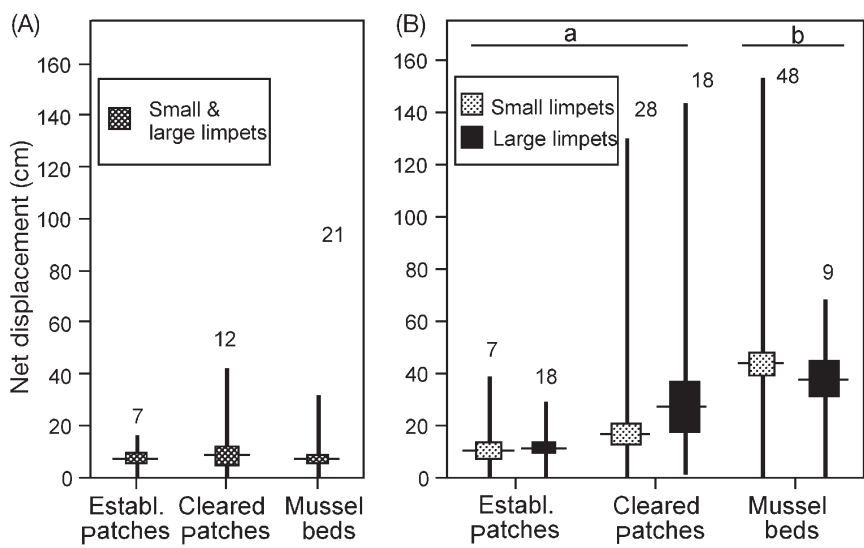

Fig. 3. Scutellastra argenvillei. Distance moved by non-homing animals in (A) $12 \mathrm{~h}$ and (B) 1 mo periods. Boxes represent 1 standard error above and below the mean. The vertical lines indicate the range of measured distances. Horizontal lines with different letters indicate significant differences (Tukey tests, $\mathrm{p}<0.01$ ) and numbers indicate sample size 
Table 2. Scutellastra argenvillei. Two-way (size, habitat) ANOVA of log-transformed data testing for differences in distances moved over a 1 mo period

\begin{tabular}{|lrccl|}
\hline Effect & df & MS effect & $F$ & $p$ \\
\hline Size & 1 & 0.706 & 0.916 & 0.34 \\
Habitat & 2 & 6.440 & 8.363 & 0.0004 \\
Interaction & 2 & 0.523 & 0.680 & 0.51 \\
Residual & 122 & 0.770 & & \\
\hline
\end{tabular}

tionship in cleared patches $(\log$ distance $=1.14+$ $0.0313 \times$ size $\mathrm{r}^{2}=0.11, \mathrm{df}=44, \mathrm{p}=0.02$ ).

In all 3 habitats, limpets moved more over 1 mo periods than on $12 \mathrm{~h}$ tidal basis (Fig. 3). Mean 1 mo distances were only slightly larger than the short-term distances in established patches, but the ratio doubled and tripled in cleared patches and on mussel beds, respectively. Thus the net effect of consecutive $12 \mathrm{~h}$ movements was a general decrease in site-specificity as limpets moved away from their original positions and did not return in later movements, and this effect was strongest for limpets on mussel beds.

\section{Movements between habitats}

Changes in the habitats occupied by Scutellastra argenvillei were studied following a 1 mo interval, after which period a total of 190 limpets could be traced. Practically all limpets remained on their initial habitat after 1 mo, except for those on mussel beds (Fig. 4). Almost one-third (29.8\%) of small limpets on mussel beds moved into cleared patches, a higher percentage than those moving from cleared patches onto mussel beds $(8.6 \%)$. Only 1 limpet ( $2 \%$ of the sample) moved out of an established patch, shifting into a neighbouring cleared patch. There were significant differences between habitats in terms of the frequency at which small limpets moved from one habitat to another $\left(\chi^{2}=11.43\right.$, $\mathrm{df}=2, \mathrm{p}=0.003$ ). Tukey-type post hoc tests indicated that the frequency of small limpets moving out of mussel beds was significantly higher than out of cleared patches $(p<0.05)$ or established patches $(p<0.01)$. Large limpets on mussel beds changed also at a slightly higher rate to another habitat compared to those in established or cleared patches. This difference was, however, not significant $\left(\chi^{2}=0.58, \mathrm{df}=2, \mathrm{p}=0.75\right)$.

As a measure of the biotic homogeneity of the 3 habitats, the average coefficients of variation were calculated for the percentage covers of the 3 most abundant sessile taxa (Mytilus galloprovincialis, Porphyra sp. and coralline turf). For established patches, mussel beds and cleared patches, the mean $( \pm 1 \mathrm{SD})$ values were, respectively, $10.6 \pm 5.2,15.2 \pm 7.0$ and $72 \pm 54 \%$.

\section{Population structure}

There were significant differences between the densities and size structure of Scutellastra argenvillei between habitats (Fig. 5). Density was greatest in established patches and least in mussel beds, with cleared patches being intermediate (ANOVA, $F_{2,12}=$ 23.37, $\mathrm{p}=0.0004$; Tukey tests, $\mathrm{p}<0.05$ between all 3 habitats). Mean size followed a similar pattern $\left(\right.$ ANOVA, $F_{2,147}=12.7, \mathrm{p}<0.001$; Tukey tests, $\mathrm{p}<0.001$ between all 3 habitats). The reduction in density in the cleared patches, and even more obviously on mussel beds, applied particularly to large animals. If the size at sexual maturity is taken to be $45 \mathrm{~mm}$ (Eekhout et al. 1992), then the decline in sexually mature limpets was dramatic: in cleared patches, sexually mature individuals were reduced to $8 \%$ of the densities in established patches, and in mussel beds they were down to $1 \%$.

\section{DISCUSSION}

Homing occurs in many limpet species and has been considered to be a behavioural response to physical stresses such as desiccation and wave action, and to biological pressures such as predation and competition (Branch 1981). Homing and movement patterns in limpets can be affected by extrinsic factors including shore height and slope, desiccation and food availability, or by intrinsic factors such as limpet size (reviewed by Branch 1981, Iwasaki 1994; also see Williams et al. 1999, Jenkins \& Hartnoll 2001). Cellana toreuma, for example, shows higher site fidelity very high on the shore, where desiccation stress is greatest, and very

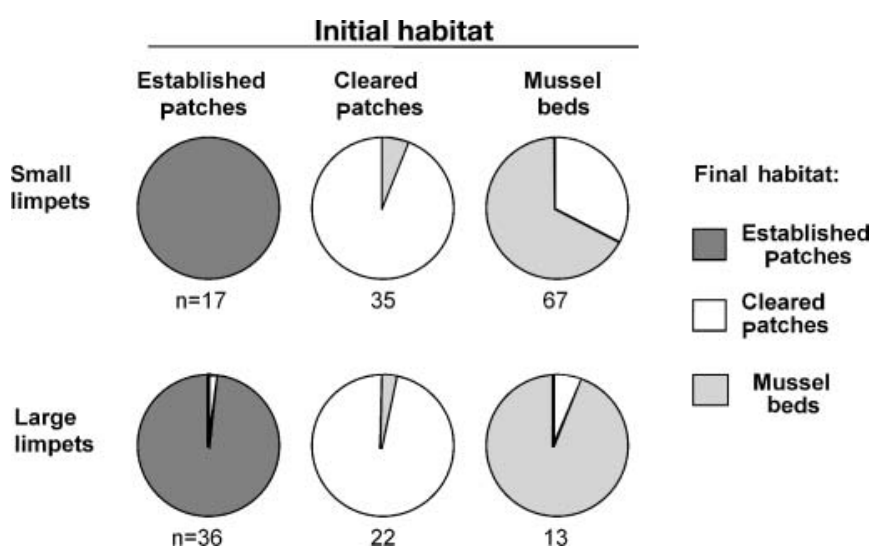

Fig. 4. Scutellastra argenvillei. Changes in habitat after $1 \mathrm{mo.}$ Figures indicate the initial number of limpets in each habitat and size class. Circle sectors represent the percentage of animals found in a different habitat after 1 mo 
A. On established patches

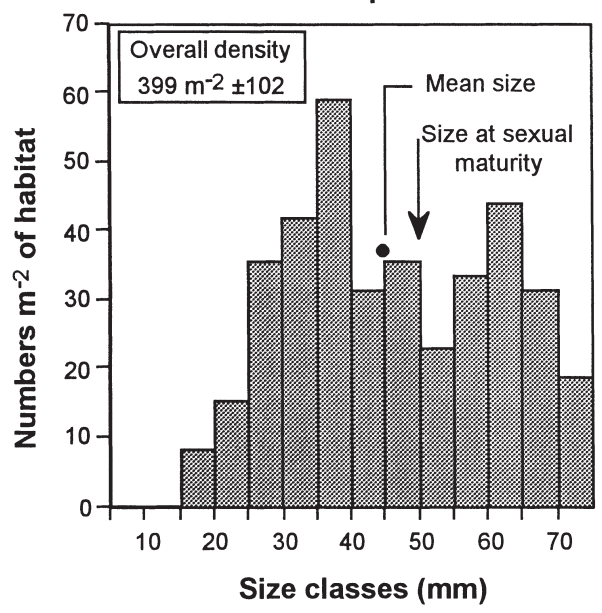

B. On cleared patches

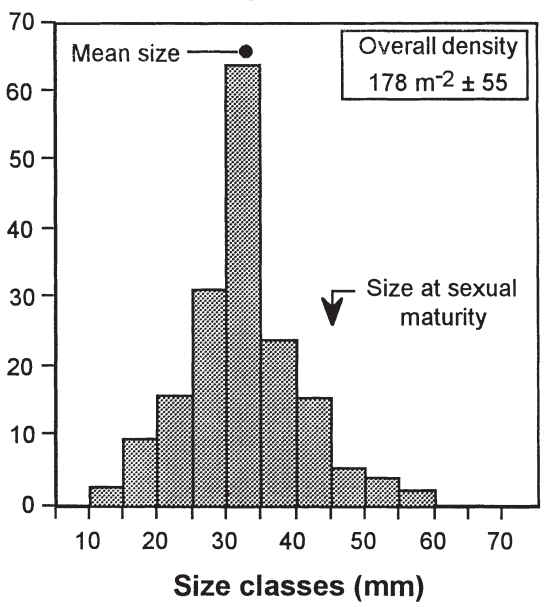

C. On mussel bed

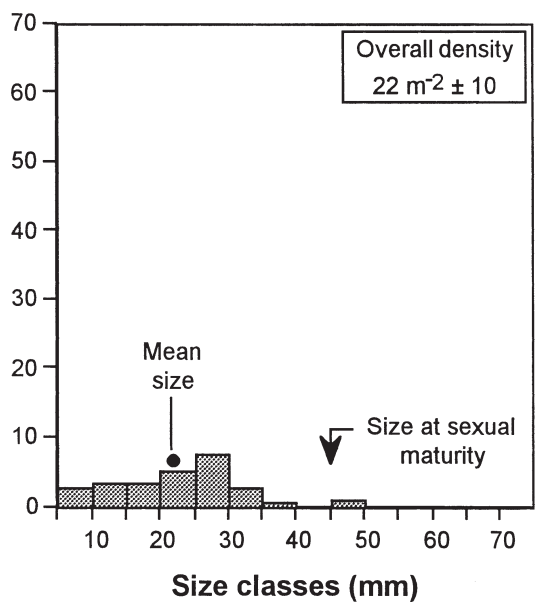

Fig. 5. Scutellastra argenvillei. Size-structure and densities on (A) established limpet patches, (B) cleared patches in mussel beds, and $(\mathrm{C})$ on beds of the mussel Mytilus galloprovincialis. The mean overall density $\mathrm{m}^{-2}( \pm 1 \mathrm{SD})$ is shown in the top right of each figure. Dots and arrows, respectively, show the mean sizes of the limpets and the size at which $50 \%$ of the population attains sexual maturity

low on the shore, where threat of predation is high; but less fidelity in the mid-shore (Iwasaki 1992). Several intertidal grazers show changes in foraging and movement patterns as a response to topographic complexity (Underwood \& Chapman 1989) or to factors affecting food distribution (Castenholz 1961, MacKay \& Underwood 1977, Chelazzi et al. 1983, Chapman \& Underwood 1992). Many grazing gastropods alter their foraging behaviour as they grow. For example, small individuals of Cymbula compressa, Scutellastra cochlear and $S$. longicosta move greater distances during foraging excursions than large ones (Branch 1971, 1975). In our study, differences between small and large $S$. argenvillei were slight. Frequencies of homing never differed. Only on cleared patches, and then only for $1 \mathrm{mo}$ and not $12 \mathrm{~h}$ data, was there a correlation between distances moved and size. Our hypothesis that the adult limpets will move less often and for shorter distances than juveniles is thus refuted.

The most clear-cut results emerging from our study were that limpets on mussel beds displayed lower frequencies of short- and medium-term homing and higher medium-term distances of movement than those in established limpet patches. Comparing 3 species of gastropods, Chapman (2000) showed that linear distances of movement tend to be greater on simple than complex surfaces. We might, therefore, have expected the movements of Scutellastra argenvillei to be hindered by mussels, but the limpets actually moved further and more frequently on these surfaces than when on topographically relatively simple rock surfaces in established or cleared patches.
Iwasaki (1999) showed that the limpet Scutellastra (= Patella) flexuosa is confined to small gaps in beds of the mussel Hormomya mutabilis. This limpet establishes fixed home sites from which it forages short distances (ca. $60 \mathrm{~mm}$ ) when awash. No individuals were ever observed on mussels, although they must be able to move across mussels because about 1 to $30 \%$ exchanged gaps each month-usually small individuals that shifted from gaps with high limpet densities to ones with lower densities. The mussel clearly restricts the amount of suitable habitat available for $S$. flexuosa. Wave-created gaps in mussel beds are vital for $S$. flexuosa.

Scutellastra argenvillei is extremely sedentary and occurs at exceptionally high densities (Bustamante et al. 1995). Large $S$. argenvillei feed collectively on fronds of living kelp (Eckonia maxima and Laminaria pallida). Experimental deprivation of kelp increased mortality of $S$. argenvillei because high-density stands of the limpet cannot subsist on in situ algal production alone. These findings suggest that the well-developed homing behaviour in $S$. argenvillei in established stands is a behavioural response to a patchy distribution of food and to the higher rate of food acquisition derived from collective feeding.

The recent invasion of exposed and semi-exposed rocky shores on the west coast of southern Africa by the Mediterranean mussel Mytilus galloprovincialis has had direct impacts on the abundance and size of Scutellastra argenvillei. However, indirect effects, such as alterations of population structure brought about by changes in foraging behaviour, are also pos- 
sible. M. galloprovincialis also outcompetes other local intertidal species for primary space (Griffiths et al. 1992, Hockey \& van Erkom Schurink 1992). Moreover, Mytilus encroachment deprives $S$. argenvillei of access to kelp, and limpets may be forced to abandon their strict homing behaviour and to search for positions where they have access to kelp, or where their densities are sufficiently low for in situ productivity to meet their needs.

Limpets in established patches (remnants of the preinvasion limpet belt) homed more consistently and moved less than limpets in other habitats. Even in the established patches, homing frequency (86.5\% over $12 \mathrm{~h}, 51.0 \%$ monthly) was lower than in natural populations that were not encroached upon by mussels, for which Bustamante et al. (1995) found that not 1 out of 507 limpets moved out of $0.5 \mathrm{~m}^{2}$ plots after 6 mo. The homing pattern was even more strikingly affected on mussel beds, where only $60 \%$ of the limpets stayed in the same position each tide and practically all limpets changed their position monthly.

Several causes for lower homing frequency and longer distances moved on mussel beds may be hypothesised, including the unsuitability of the substratum for the formation of a home scar, the irregular topography of the mussel matrix or, in the case of large limpets, space limitations caused by the size and shape of the mussels. Other causes may involve difficulties in forming a scar, laying down a chemical trail, or following the trail back. Another hypothesis may be based on a behavioural shift induced by inaccessibility to subtidal kelp. Limpets on mussel beds may move more because they are deprived of prime sites for passive access to living kelp and need to change their feeding strategy and actively search for kelp or alternative food sources. Limpets on mussel beds did not cluster (reaching densities of only 10 to 30 limpets $\mathrm{m}^{-2}$ of mussel bed), whereas those on established patches were densely aggregated (ca. $400 \mathrm{~m}^{-2}$ of habitat). The observed frequency and extent of limpet movements on mussel beds would make aggregations within reach of subtidal kelp a fortuitous event. 'Mushrooming' (elevation of the shell) to trap kelp blades (Bustamante et al. 1995) was never observed in limpets on mussel beds, and the efficiency of this method of feeding on a substratum as irregular as the mussel beds is questionable.

Although homing frequency was not significantly different between large and small limpets in any habitat, it was lower on mussel beds than other substrata, and lower on cleared patches than established patches. Large limpets in established patches were observed forming clusters at the edge of the patch, where living kelp fronds were brought within reach by waves, and small limpets were found between or on the shells of adults. For animals that rely substantially on kelp and benefit from collective feeding, accessibility to this source of food would favour a strict homing behaviour ('sit-and-wait' strategy), which would be less meaningful for small animals with a more generalist diet. On mussel beds, however, where in situ food is scarcer and uniformly distributed, and capture of kelp blades may even be impossible, large limpets would derive little benefit from strict homing and may be forced to actively search ('free-ranging' strategy) for alternative food sources, including drift kelp. Small limpets, on the other hand, with a more generalist diet and lower absolute energetic demands may be able to obtain their food supplies via short excursions in the mussel beds.

Limpets in established patches almost all homed to a specific position, and limpets on mussel beds nearly all moved frequently. By contrast, limpets in cleared patches showed more variability in homing and movement patterns, possibly related to the more heterogeneous conditions of food type and availability, substratum and density experienced there. Comparable shifts in feeding behaviour related to spatial variability in food availability have been reported elsewhere. Mackay \& Underwood (1977) found that the limpet Cellana tramoserica homed more frequently where food was abundant, changing to free-ranging behaviour when food was scarce. Studying two sympatric species of chiton, Chelazzi et al. (1983) found that animals remained in a feeding location when food was distributed in patches (cf. established patches) whereas site-specificity was lower when food was uniformly distributed (cf. mussel beds, where micro-algal distribution is probably more homogeneous).

Chapman \& Underwood (1992) reviewed the types of foraging behaviour of benthic grazers, classifying them as free-ranging foragers, foragers in a patchily distributed food source, foragers in a patchy distribution of shelter, and sit-and-wait grazers. Scutellastra argenvillei in its natural habitat, and in the established patches of this study, behaves as a sit-and-wait grazer, but changes to a free-ranging or food-patchiness forager when on mussel beds. Small limpets may be closer to the free-ranging end of this continuum than large limpets, because of their more generalist diet. Chapman \& Underwood (1992) note that many limpets free-range when small and become territorial (and, presumably, more specialised) when larger.

The population structure of Scutellastra argenvillei was radically different in the 3 habitats examined. Eekhout et al. (1992) recorded a modal shell length of $75 \mathrm{~mm}$ for $S$. argenvillei before its populations were encroached by Mytilus galloprovincialis, in populations equivalent to what we termed 'established patches'. In the present study, limpets larger than $70 \mathrm{~mm}$ did occur in established patches, but the mean size was only $45 \mathrm{~mm}$. In cleared patches, no limpets 
over $65 \mathrm{~mm}$ were recorded and mean size was $34 \mathrm{~mm}$. Few limpets larger than $50 \mathrm{~mm}$ were found on mussel beds, the largest being $59 \mathrm{~mm}$, and the mean size was only $21 \mathrm{~mm}$. In parallel with this, large limpets on mussel beds had the lowest rate of recapture at the end of the study, implying a high mortality rate. It is likely that large limpets on mussel beds had to rely on a less nutritious diet than usual and were forced into longer feeding excursions, and thus greater energetic expenditure. The effects of this are likely to be even greater than estimated by our observations because limpet movements are more tortuous on complex substrata than on smooth surfaces (Erlandsson et al. 1999). Thus, in absolute terms, the differences between distances moved on complex surfaces such as mussel beds versus smooth rocks will be even greater than our estimates based on linear displacements. The irregularity of the mussel-bed surface, the probability that the convex shape of the mussel shells will reduce the efficacy with which the limpet can clamp against the substratum, and the fact that the limpets fail to form homescars on the mussels may conspire to increase the vulnerability of mussel-dwelling limpets to predation or dislodgement by wave action, thus increasing mortality rates. Hockey \& van Erkom Schurink (1992) found a significant positive correlation between the lengths of $S$. granularis and the lengths of the mussels they occupy, and concluded that the maximum size attainable by limpets in mussel beds is limited by the size of individual mussels.

Small limpets were proportionally more abundant than large ones on mussel beds, but even so, their absolute densities there were much less than those in other habitats. By virtue of their smaller size, they can enter the mussel matrix, finding shelter from desiccation and wave action. The mussel matrix, with its intricate 3-dimensional layout, slows down water flow, which may favour settlement or enhance post-settlement survival of the limpets due to reduced desiccation. Limpet species that never reach a large size relative to mussels may readily find a permanent habitat on the shells of mussels, as is the case for Scutellastra aphanes, which lives almost exclusively on the mussel Perna perna (Robson 1986). S. granularis settles prolifically in beds of Mytilus galloprovincialis, but large individuals become excluded and cannot survive there (Hockey \& van Erkom Schurink 1992). As S. granularis becomes sexually mature at a relatively small size, the high densities of small reproductive individuals in mussel beds generate a total reproductive output per unit area greater than is accomplished by low-density, larger individuals occupying bare rock that lacks mussels (Griffiths et al. 1992). The lifetime per capita reproductive output of individuals is, however, lower in mussel beds.
For Scutellastra argenvillei, the situation is more parlous. It is much larger and reaches sexual maturity at about $45 \mathrm{~mm}$ (Eekhout et al. 1992). In mussel beds, large individuals appear to have a low survival rate, as reflected in the density and size-composition data. Few reproductive individuals occur on mussel beds. Mytilus galloprovincialis therefore not only alters the homing and movement behaviour of $S$. argenvillei, but preempts space that it would normally occupy, reduces its access to the kelp needed to sustain high-density populations of the limpet, and poses a direct threat to its reproductive output.

There have been frequent proposals to commercially harvest Scutellastra argenvillei and another limpet, Cymbula (= Patella) granatina on the west coast of South Africa (Eekhout et al. 1992). Mytilus galloprovincialis does not interact with C. granatina, which predominates in sheltered boulder-bays, where the mussel is scarce (Bustamante et al. 1995). In the case of S. argenvillei, however, the effects of the mussel are troubling in the light of applications for commercial harvesting of the limpet. $S$. argenvillei may be able to maintain itself in established patches, but these are quickly dominated by the mussel if the limpets are experimentally thinned or removed (Eekhout et al. 1992). Furthermore, settlement and recruitment of $M$. galloprovincialis is greatly increased in patches from which $S$. argenvillei has been cleared (Steffani 2001). Harvesting will thus speed up competitive displacement of $S$. argenvillei by the mussel, and further reduce the availability of the primary substratum so necessary for the limpet to reach a size at which it can achieve sexual maturity.

Summarising, mussel encroachment on belts of Scutellastra argenvillei induces behavioural changes that result in decreased homing and increased frequency and distance of movement, thus shifting the foraging strategy of the limpets from a 'sit-and-wait' approach in established patches to a 'free-ranging' strategy on mussel beds. The behavioural pattern is less defined in cleared patches, probably due to greater variability in the physical and biological characteristics of this habitat. Limpet population structure is also affected by mussel encroachment, with a reduction in mean limpet size and density, and dramatically fewer reproductive individuals on mussel beds. The immigration dynamics of limpets on cleared patches is one aspect of considerable importance for the future of communities encroached by Mytilus galloprovincialis, as these patches may act as temporary refugia and provide opportunities for $S$. argenvillei to survive the limitations imposed by this alien mussel. Thus, apart from the obvious effects of space occupation by M. galloprovincialis, this mussel has subtle effects on the behaviour of $S$. argenvillei, and these indirectly impact on survivorship and reproductive output. 
Acknowledgements. Funding for this research was provided by the National Research Foundation (NRF), the Mellon Foundation, the University of Cape Town (UCT), and the South African Network for Coastal and Oceanographic Research (SANCOR). We appreciate the work put in by 4 anonymous reviewers to improve the paper.

\section{LITERATURE CITED}

Branch GM (1971) The ecology of Patella Linnaeus from the Cape Peninsula, South Africa. I. Zonation, movements and feeding. Zool Afr 6:1-38

Branch GM (1975) Mechanisms reducing intraspecific competition in Patella spp.: migration, differentiation and territorial behaviour. J Anim Ecol 44:575-600

Branch GM (1976) Interspecific competition experienced by South African Patella species. J Anim Ecol 45:507-529

Branch GM (1978) The response of South African patellid limpets to invertebrate predators. Zool Afr 13:221-232

Branch GM (1981) The biology of limpets: physical factors, energy flow and ecological interactions. Oceanogr Mar Biol Annu Rev 19:235-380

Branch GM (1984) Competition between marine organisms: ecological and evolutionary implications. Oceanogr Mar Biol Annu Rev 22:429-593

Branch GM (1988) Activity rhythms in Siphonaria thersites. In: Chelazzi G, Vannini M (eds) Behavioural adaptation to intertidal life. NATO ASI Series, Vol 151. Plenum Press, New York, p 27-44

Branch GM, Cherry MI (1985) Activity rhythms of the pulmonate limpet Siphonaria capensis Q. and G. as an adaptation to osmotic stress, predation and wave action. J Exp Mar Biol Ecol 87:153-168

Bustamante RH, Branch GM, Eekhout S (1995) Maintenance of an exceptional intertidal grazer biomass in South Africa: subsidy by subtidal kelps. Ecology 76(7):2314-2329

Castenholz RW (1961) The effect of grazing on marine littoral diatom populations. Ecology 42:783-794

Chapman MG (2000) A comparative study of differences among species and patches of habitat on movements of three species of gastropods. J Exp Mar Biol Ecol 22:181-201

Chapman MG, Underwood AJ (1992) Foraging behaviour of marine benthic grazers. In: John DM, Hawkins SJ, Price JH (eds) Plant-animal interactions in the marine benthos. Systematics Association Special, Vol 46. Clarendon Press, Oxford, p 289-317

Chelazzi G (1990) Eco-ethological aspects of homing behaviour in molluscs. Ethol Ecol Evol 2:11-26

Chelazzi G, Focardi S, Deneubourg JL (1983) A comparative study on the movement patterns of two sympatric tropical chitons (Mollusca: Polyplacophora). Mar Biol 74:115-126

Chelazzi G, Focardi S, Deneubourg JL (1988) Analysis of movement patterns and orientation mechanisms in intertidal chitons and gastropods. In: Chelazzi G, Vannini M (eds) Behavioural adaptation to intertidal life. NATO ASI Series, Vol 151. Plenum Press, New York, p 173-184

Cook A, Bamford OS, Freeman JDB, Teideman DJ (1969) A study of the homing habit of the limpet. Anim Behav 17: 330-339

Creese RG (1982) Distribution and abundance of the acmaeid limpet Patelloida latistrigata and its interaction with barnacles. Oecologia 52:85-96

Eekhout S, Raubenheimer CM, Branch GM, Bosman AL, Bergh MO (1992) A holistic approach to the exploitation of intertidal stocks: limpets as a case study. S Afr J Mar Sci 12:1017-1029
Erlandsson J, Kostylev V, Williams GA (1999) A field technique for estimating the influence of surface complexity on movement tortuosity in the tropical limpet Cellana grata Gould. Ophelia 50:215-224

Funke W (1968) Heimfindevermögen und Ortstreue bei Patella L. (Gastropoda: Prosobranchia). Oecologia 2:19-142

Garrity SD, Levings SC (1983) Homing to scars as a defence against predators in the pulmonate limpet Siphonaria gigas (Gastropoda). Mar Biol 72:319-324

Geller JB (1999) Decline of a native mollusc masked by a sibling species invasion. Conserv Biol 13:661-664

Grant WS, Cherry MI (1985) Mytilus galloprovincialis Lmk. in southern Africa. J Exp Mar Biol Ecol 90:179-191

Gray DR, Hodgson AN (1998) Foraging and homing behaviour in the high-shore, crevice-dwelling limpet Helcion pectunculus (Prosobranchia: Patellidae). Mar Biol 132: 283-294

Griffiths CL, Hockey PAR, van Erkom Schurink C, le Roux PJ (1992) Marine invasive aliens of South African shores: implications for community structure and trophic functioning. S Afr J Mar Sci 12:713-722

Hartnoll RG (1985) The monitoring of limpet movement: a review. Progr Underwater Sci 11:137-146

Hawkins SJ, Hartnoll RG (1983) Grazing of intertidal algae by marine invertebrates. Oceanogr Mar Biol Annu Rev 21: 195-282

Hockey PAR, van Erkom Schurink C (1992) The invasive biology of the mussel Mytilus galloprovincialis on the southern African Coast. Trans R Soc S Afr 48:123-139

Iwasaki K (1992) Factors affecting individual variation in resting site fidelity in the patellid limpet Cellana toreuma (Reeve). Ecol Res 7:305-331

Iwasaki K (1993a) Analyses of limpet defence and predator offense in the field. Mar Biol 116:277-289

Iwasaki K (1993b) Synergistic effects of mixed grazing by intertidal limpets on sessile organisms: consequences of differences in grazing ability and feeding habit. Physiol Ecol Jpn 30:1-30

Iwasaki K (1993c) The role of individual variability in limpet resting site fidelity and competitive ability in the organisation of a local rocky intertidal community. Physiol Ecol Jpn $30: 31-70$

Iwasaki K (1994) Intra- and interspecific variation in activity patterns of intertidal limpets. Venus Jpn J Malacol 53: 85-104

Iwasaki K (1995) Dominance order and resting site fidelity in the intertidal pulmonate limpet Siphonaria sirius (Pilsbry). Ecol Res 10:105-115

Iwasaki K (1999) Short- and long-term movements of the patellid limpet Patella flexuosa within gaps in intertidal mussel beds. J Molluscan Stud 5:295-301

Jenkins SR, Hartnoll RG (2001) Food supply, grazing activity and growth rate in the limpet Patella vulgata L.: a comparison between exposed and sheltered shores. J Exp Mar Biol Ecol 258:123-139

Lewis JR, Bowman RS (1975) Local habitat induced variations in the population dynamics of Patella vulgata L. J Exp Mar Biol Ecol 17:165-203

Little C (1989) Factors governing patterns of foraging activity in littoral marine herbivorous molluscs. J Molluscan Stud 55:273-284

Mackay DA, Underwood AJ (1977) Experimental studies on homing in the intertidal patellid limpet Cellana tramoserica (Sowerby). Oecologia 30:215-237

McDonald JH, Koehn RK (1988) The mussels Mytilus galloprovincialis and $M$. trossulus on the Pacific coast of North America. Mar Biol 99:111-118 
Paine RT, Levin SA (1981) Intertidal landscapes: disturbance and the dynamics of pattern. Ecol Monogr 51(2):145-178

Ridgway SA, Reid DG, Taylor JD, Branch GM, Hodgson AN (1998) A cladistic phylogeny of the family Patellidae (Mollusca: Gastropoda). Phil Trans R Soc Lond B 353: 1645-1671

Robson G (1986) A new species of South African limpet, Patella aphanes (Mollusca: Gastropoda: Patellidae), with a discussion of P. obtecta Krauss, 1869. Durban Mus Novit 13:305-320

Serra G, Chelazzi G, Castilla JC (2001) Temporal and spatial activity of the key-hole limpet Fissurella crassa (Mollusca: Gastropoda) in the eastern Pacific. J Mar Biol Assoc UK 81:485-490

Steffani CN (2001) Interactions between an indigenous limpet, $S$. argenvillei, and an alien invasive mussel, Mytilus galloprovincialis: moderation by wave action. PhD thesis, University of Cape Town

Stephenson A (1939) The constitution of the intertidal fauna and flora of South Africa. Part I. J Zool 4025:487-533

Stimson J (1970) Territorial behaviour of the owl limpet Lottia gigantea. Ecology 51:113-118

Editorial responsibility: Roger Hughes (Contributing Editor), Bangor, Wales, UK
Tokeshi M, Romero L (1995) Filling a gap: dynamics of space occupancy on a mussel-dominated subtropical rocky shore. Mar Ecol Prog Ser 119:167-16

Underwood AJ (1977) Movements of intertidal gastropods. J Exp Mar Biol Ecol 26:191-201

Underwood AJ (1979) The ecology of intertidal gastropods. Adv Mar Biol 16:111-210

Underwood AJ (1997) Experiments in ecology. Cambridge University Press, Cambridge

Underwood AJ, Chapman MG (1989) Experimental analyses of the influences of topography of the substratum on movements and density on an intertidal snail, Littorina unifasciata. J Exp Mar Biol Ecol 134:175-196

van Erkom Schurink C, Griffiths CL (1990) Marine mussels of Southern Africa - their distribution patterns, standing stocks, exploitation and culture. J Shellfish Res 9(1):75-85

Williams GA, Little C, Morritt D, Stirling P, Teagle L, Miles A, Pilling G, Consalvey $M$ (1999) Foraging in the limpet Patella vulgata: the influence of rock slope on the timing of activity. J Mar Biol Assoc UK 79:881-889

Zar JH (1999) Biostatistical analysis. Prentice-Hall, Englewood Cliffs, NJ

Submitted: August 17, 2001; Accepted: June 11, 2002 Proofs received from author(s): October 18, 2002 